\title{
THE MAGNETIC FIELDS IN THE ENVELOPES OF PROTO-PLANETARY NEBULAE
}

\author{
J.Y. HU \\ Beijing Observatory, Academia Sinica, Beijing, 100080, China
}

It is possible that the magnetic field plays important role in the formation of planetary nebulae(Poscoli,1992). In order to measure the strength of magnetic field in the envelope of protoplanetary nebulae(PPNe) we have used the Max-Planck-Institut fur Radioastronomie 100-m telescope at Effelsberg to obtain the high frequency resolution and high signal-to-noise ratio $1612 \mathrm{MHz}$ spectra of PPNe, IRAS08005-2356, 18276-1431, and 20406+2953 in both circular polarization. The nature of PPN of these objects are confirmed by Slikhuis et al.(1991), Le Bertre(1987), and Hu et al.(1992) based on the extensive optical, infrared and radio molecular line observations.

The OH maser from IRAS08005 shows multi-peak structure and the Zeeman splitting is larger than width of individual peaks. We can easyly find the Zeeman split pairs with $\delta \mathrm{V}=0.81 \mathrm{~km} / \mathrm{sec}$. It results the strength of magnetic field of $2.3 \mathrm{mG}$. But we also found that some peaks are without counterparts. It means that in the masing region the magnetic field is not uniform.

In the case of IRAS18276 and 20406 the Zeeman splitting is smaller than width of $\mathrm{OH}$ maser peaks. The Stokes parameters of $I(\nu)$ and $V(\nu)$ are used to derive the strength of field. It results 0.33 and $0.26 \mathrm{mG}$. It is also clearly shown that not all of components of peaks are polarized. So the results are the low-limited values. Again this confirms that the magnetic field in the masing region in these two objects are not uniform.

The strength of magnetic fields in the masing region of PPNe are considerably stronger than that in the envelopes of OH/IR stars found by Zell and Fix(1991) on the order of 1-100 $\mu \mathrm{G}$.

Hu,J. Y. et al.,1992, in preparing.

Le Bertre, T., 1987, A\&A, 180,160.

Pascoli, G.,1992, PASP,104, 350.

Slijkhuis, S. et al.,1991, A\&A,248, 547.

Zell, P. J., Fix, J. D.,1991, ApJ,369,506. 